Revue des patrimoines

46 | 2022

Le patrimoine de la Justice

\title{
L'École nationale de la magistrature : une architecture nouvelle pour une justice rénovée (1960-1972)
}

The French National School for the Judiciary: a new architecture for a renewed justice (1960-1972)

\section{Franck Delorme}

\section{OpenEdition}

Journals

Édition électronique

URL : https://journals.openedition.org/insitu/33330

DOI : 10.4000/insitu.33330

ISSN : $1630-7305$

Éditeur

Ministère de la Culture

\section{Référence électronique}

Franck Delorme, «L'École nationale de la magistrature : une architecture nouvelle pour une justice rénovée (1960-1972) », In Situ [En ligne], 46 | 2022, mis en ligne le 10 janvier 2022, consulté le 03 février 2022. URL : http://journals.openedition.org/insitu/33330 ; DOl : https://doi.org/10.4000/insitu 33330

Ce document a été généré automatiquement le 3 février 2022.

\section{(c) $(1) \odot$}

In Situ Revues des patrimoines est mis à disposition selon les termes de la licence Creative Commons Attribution - Pas d'Utilisation Commerciale - Pas de Modification 4.0 International. 


\title{
L'École nationale de la
} magistrature : une architecture nouvelle pour une justice rénovée (1960-1972)

\author{
The French National School for the Judiciary: a new architecture for a renewed \\ justice (1960-1972)
}

Franck Delorme

Dans le centre de Bordeaux, un îlot entier est dédié à la Justice. Dans un quadrilatère délimité par la place de la République, le cours d'Albret, la rue du Maréchal-Joffre et la rue des Frères-Bonie sont regroupés trois édifices qui, par l'époque de leur construction et donc leur architecture, incarnent chacun à leur manière la fonction judiciaire. Entre le palais de justice du milieu du xIX ${ }^{\mathrm{e}}$ siècle et le tribunal de grande instance de la toute fin du $\mathrm{xx}^{\mathrm{e}}$ siècle, l'École nationale de la magistrature représente, par sa fonction de formation des magistrats, la transition entre la justice du passé et celle d'aujourd'hui [fig. 1]. Les bâtiments de l'École nationale de la magistrature marquent aussi le renouvellement de l'architecture judiciaire tel qu'il a été voulu dès le lendemain de la Seconde Guerre mondiale et mis en œuvre durant les décennies qui ont suivi. Cette architecture reste cependant singulière par son programme unique et par le caractère que lui a donné son architecte, Guillaume Gillet (1912-1987), un acteur principal de l'architecture française des Trente Glorieuses. 
Figure 1

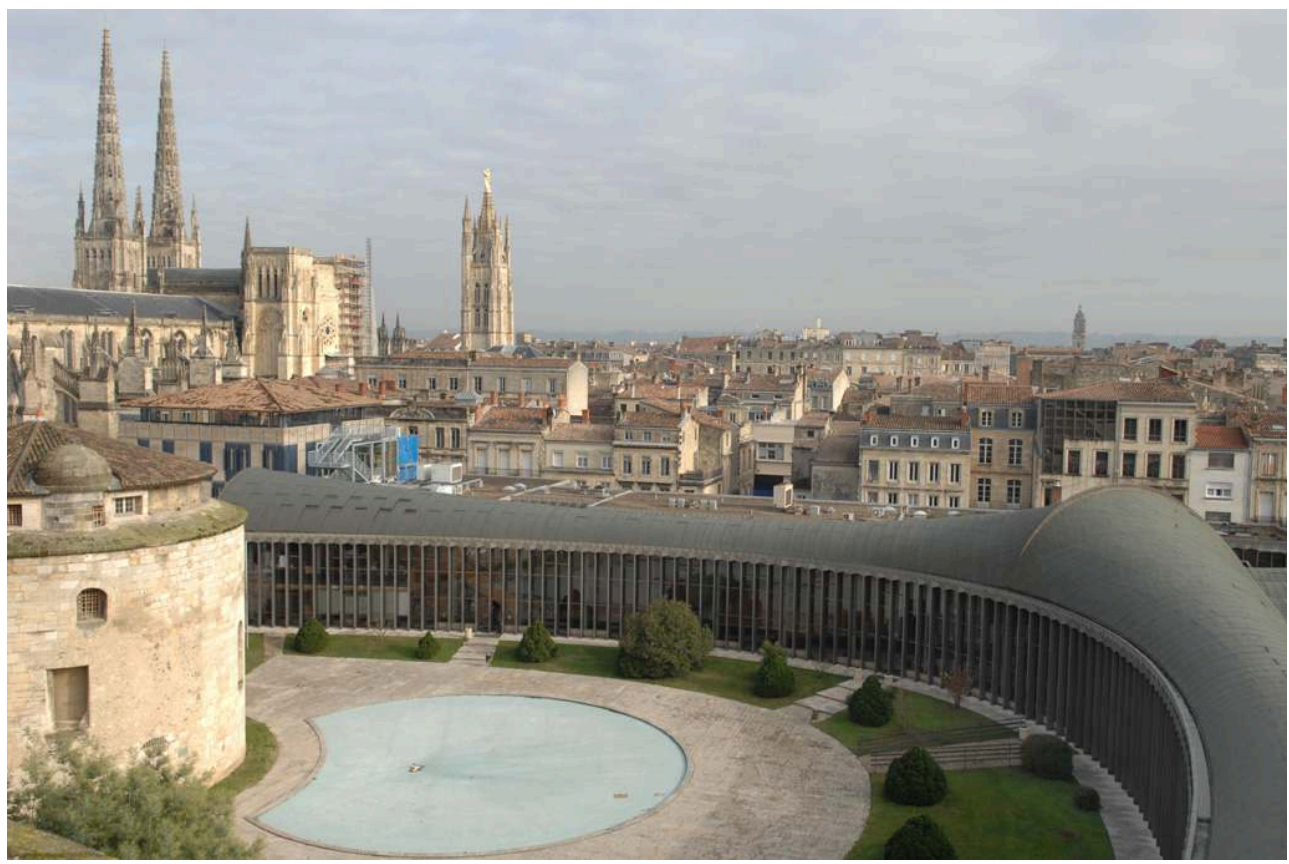

La cour de l'École nationale de la Magistrature avec à l'arrière-plan les tours et les toitures de la cathédrale Saint-André.

(c) Jérémy Buchholz / @ Guillaume Gillet.

\section{Une école d'un nouveau type : une «ENA des magistrats »}

2 Dès 1945, il fut envisagé la création d'une section judiciaire au sein de l'École nationale d'administration tout juste fondée. Plus d'une décennie plus tard, l'idée d'une institution dédiée spécifiquement à la formation des magistrats resurgit lors de l'élaboration de la réforme de la justice. Dans le cadre de la rédaction de la Constitution de la Ve République, le garde des Sceaux Michel Debré porta «la volonté de refaire les institutions de la République, mais tout autant de relever le corps de la magistrature et si possible d'y ajouter une remise en ordre de l'organisation judiciaire et de la procédure ». Cette réforme passait par la création d'un «Centre national d'études judiciaires destiné au recrutement d'un corps unique de magistrats ${ }^{1} »$.

$3 \mathrm{Au}$ sein du corpus de textes qui mirent en place, entre autres choses, la réorganisation des juridictions ou l'application du Code de procédure pénale, une ordonnance instituait le Centre national d'études judiciaires ${ }^{2}$ (CNEJ). Rattaché au ministère de la Justice et placé sous l'autorité du garde des Sceaux ${ }^{3}$, ce nouvel établissement d'enseignement supérieur avait donc pour missions l'organisation des concours d'accès à la magistrature, la formation initiale et la formation continue de l'ensemble des magistrats français, la contribution à la formation de futurs magistrats de pays liés à la France par des accords de coopération, et enfin le perfectionnement des magistrats de ces mêmes États ${ }^{4}$.

4 D'une durée de trente-et-un mois, la formation initiale devait privilégier la pratique par la simulation d'audiences, le travail en ateliers, les stages, au cours desquels les 
auditeurs de justice 医 nom donné aux étudiants théorie et la méthodologie enseignées à la pratique professionnelle et aux réalités sociales, économiques et humaines. L'objectif des études était l'acquisition des compétences fondamentales transversales et communes à tous les métiers de la magistrature, fondées sur l'éthique, la déontologie, l'écoute, la conduite d'entretiens, la décision et l'acte de juger.

5 La création du CNEJ représentait un changement sans précédent, faisant passer «la formation des magistrats de l'apprentissage sur le tas à l'école professionnelle ${ }^{5}$ » et aboutissant, d'une organisation provinciale disparate, à une centralisation unificatrice.

\section{L'implantation de l'École de la magistrature à Bordeaux, un choix politique}

6 D'abord logé dans les locaux du ministère de la Justice place Vendôme à Paris, le CNEJ fut rapidement installé dans un hôtel particulier rue de la Faisanderie dans le $16^{\mathrm{e}}$ arrondissement. En 1960, la décision fut prise de transférer l'établissement à Bordeaux. Jacques Chaban-Delmas (1915-2000), alors maire de Bordeaux et député de la $2^{e}$ circonscription de la Gironde, et président de l'Assemblée nationale depuis décembre 1958, ancien ministre, joua un rôle important dans cette décision. Il conduisait alors un programme de grands travaux avec pour ambition de faire de Bordeaux une des grandes métropoles régionales, rivalisant avec Marseille, Lyon, Lille ou Strasbourg.

7 Le projet rejoignait la volonté gouvernementale de décentralisation des grands établissements publics, notamment ceux d'enseignement supérieur. C'est ainsi qu'il fut procédé au redéploiement et à la création d'établissements de formation des fonctionnaires. La construction de ces nouveaux établissements d'enseignement supérieur et de formation fut confiée à des architectes, pour la plupart membres du corps des Bâtiments civils et Palais nationaux (BCPN). À Bernard de La Tour d'Auvergne (1923-1976) furent confiées l'École nationale des impôts à Clermont-Ferrand (Puy-deDôme, 1974) et l'École nationale des services du Trésor à Noisiel (Seine-et-Marne, 1978). Grand Prix de Rome en 1944, Henri Pottier (1912-2000) fut chargé en 1965 de la construction de la nouvelle École polytechnique à Palaiseau (Essonne), inaugurée en 1976.

8 Comme le déplorait le garde des Sceaux René Pleven (1901-1993) en 1972, lors de l'inauguration de l'École nationale de la magistrature, «le déplacement de grandes écoles vers la province se heurt(a) sans conteste à beaucoup d'habitudes prises, et même de parti pris » alors qu'il était nécessaire et utile " de mettre fin, dans le domaine de la culture et de la vie intellectuelle, au parisianisme trop exclusif» et «à la concentration dans la capitale [des] plus hauts établissements d'enseignement et de tous les moyens d'information et de recherche qui les accompagnent ${ }^{6} »$. On réalise alors ce qu'avait pu représenter la création et l'implantation à Bordeaux de l'École de la magistrature douze ans auparavant. 


\section{Élaborer un programme et un concevoir un projet}

9 Afin d'assurer l'accueil de la première promotion d'auditeurs de justice en 1960, le CNEJ fut installé à Bordeaux de façon provisoire dans le quartier des Chartrons, au 17 cours Xavier-Arnozan, dans un immeuble acheté par la Ville de Bordeaux et mis à disposition du ministère de la Justice. Ces locaux étant trop petits, une grande partie des services resta à Paris, symbole de la réticence à l'exil en province, et les salles d'audience du palais de justice, élevé de 1839 à 1846 par l'architecte Adolphe Thiac (1800-1865), accueillirent les cours et les exercices pratiques des élèves-magistrats. La distance entre les deux sites - deux kilomètres -imposait de traverser tout le centre-ville pour se rendre de l'un à l'autre. Cette situation précaire et inconfortable rendait urgente la nécessité de confier à un architecte la conception des futurs bâtiments du CNEJ.

Il était donc indispensable de définir ce que devait être le lieu de formation des futurs magistrats (juges et procureurs). Ce ne devait pas être, à proprement parler, un banal établissement dans lequel serait dispensé un traditionnel enseignement supérieur spécialisé, encore moins « une école au sens strict du terme, ni un moule produisant des magistrats standard ${ }^{7}$ (sic) ». Le CNEJ devait davantage être pensé comme école d'application destinée à mettre en situation les futurs magistrats. Le lieu devait ainsi permettre les échanges et les confrontations, favoriser les rencontres et les débats. Plus que des salles de cours, les besoins consistaient davantage en espaces de réunion et en une bibliothèque-documentation mettant à disposition une connaissance la plus large possible dans tous les domaines, pour que les auditeurs y puisent la matière nécessaire pour étayer leurs décisions et jugements.

11 Le modèle de cet établissement d'un genre nouveau se rapprochait davantage de ce que son architecte, Guillaume Gillet (1912-1987), a défini comme "une sorte d'académie socratique où, par équipes de 10 à 12 , sous la direction de maîtres de conférences, des études, livres en main, sont conduites quotidiennement en petit comité, et quotidiennement menées des conversations d'un groupe à l'autre, d'un élève à l'autre ${ }^{8} "$.

12 Le programme soumis à l'architecte était donc d'une grande simplicité et assez éloigné de ceux généralement plus complexes des établissements d'enseignement supérieur, notamment dans les domaines scientifiques et techniques, comme les écoles d'ingénieurs ou les instituts de recherche universitaires.

\section{Guillaume Gillet, architecte au service de la Justice}

13 En 1960, la commande directe étant la règle, les administrations d'État avaient plusieurs options lorsqu'elles avaient besoin de recourir aux services de maitres d'œuvre pour la construction ou l'entretien de leurs bâtiments. Les grands ministères employaient des architectes conseils qui, tout en conservant une activité libérale, pouvaient mener des expertises, définir des programmes d'entretien ou de construction, examiner des projets, voire dans certains cas, être également chargés de la conception d'équipements.

14 Les administrations centrales disposaient aussi d'un vivier de professionnels dans lequel elles pouvaient puiser, en particulier lorsqu'elles souhaitaient mettre en chantier un nouveau bâtiment pour abriter leurs services. Sous la tutelle du ministère 
des Beaux-Arts, puis de celui des Affaires culturelles, elles s'adressaient alors à un architecte des Bâtiments civils et Palais nationaux (BCPN). La commande publique d'État était ainsi, dans sa grande majorité, dévolue aux membres de ce groupe, recrutés parmi les architectes titulaires du Prix de Rome ou cooptés.

Guillaume Gillet remplissait toutes les conditions pour être chargé par le ministère de la Justice du Centre national d'études judiciaires. Titulaire du Prix de Rome en 1946, il était architecte conseil du ministère de la Justice depuis 1954, désigné conjointement avec son collègue Claude Charpentier (1909-1995) pour remplir deux missions importantes. La première visait la rénovation du parc immobilier pénitentiaire par un renouvellement architectural des établissements dont Gillet fut l'acteur majeur durant une vingtaine d'années en construisant une dizaine de prisons dont celle de FleuryMérogis ${ }^{9}$ (Essonne).

16 La seconde mission portait sur le suivi des travaux d'entretien, d'aménagement et d'extension, voire de reconstruction, des palais de justice suite notamment à la distinction faite entre tribunaux d'instance et de grande instance introduite par la réforme judiciaire de 1958.

17 La réputation de Guillaume Gillet ne lui venait pas tant de ces travaux pour le ministère de la Justice que de deux réalisations ayant marqué, elles aussi, un renouvellement architectural dans des domaines bien différents. Au cœur de la ville de Royan (Charente-Maritime), anéantie par les bombardements de 1944-1945, l'église NotreDame offrit en 1958 un aspect radicalement différent de l'architecture religieuse, symbolisant ainsi le renouveau de l'art sacré ${ }^{10}$ grâce en particulier à l'usage du béton armé et du procédé des voiles à double courbure de l'ingénieur Bernard Laffaille (1900-1955). La même année, un des clous de l'Exposition universelle de Bruxelles fut le pavillon de la France, tout autant geste architectural que prouesse technique ${ }^{11}$. Ces deux réalisations ayant contribué à la renommée de Gillet, il était donc naturel et légitime de s'adresser à lui pour concevoir un des éléments essentiels dans lequel allait s'incarner la nouvelle Justice.

18 La désignation de Gillet est aussi peut-être venue compenser son échec en 1957 au concours pour la reconstruction du palais de justice de Lille (Nord), le premier chantier de ce type après 1945. Gillet y avait proposé une reconstruction par phases, le nouveau bâtiment venant remplacer progressivement l'ancien, dans une sorte de transposition moderne de l'image monumentale de l'institution.

\section{Atouts et contraintes de la situation et du terrain}

19 L'architecte désigné, le programme à peu près défini, il restait à choisir un emplacement pour édifier la future école des magistrats. Bordeaux étant le siège d'une des plus grandes juridictions pénales de France, le choix du terrain revêtait donc une importance toute particulière. Il fut décidé que le CNEJ serait implanté dans le centreville, à proximité immédiate du palais de justice. Le terrain retenu était celui de la prison, élevée au xix siècle par Thiac en même temps que le palais et mitoyenne de celui-ci.

Ce fut donc à la faveur du projet de transfert de la prison vers Gradignan, dans la banlieue sud de Bordeaux, dans une nouvelle maison d'arrêt dont la construction fut aussi confiée à Gillet, que put être résolue la question de l'implantation du futur 
établissement. Ce transfert avait pour cadre la réforme entreprise par l'administration qui prévoyait l'éloignement des lieux d'enfermement des centres-villes vers leurs périphéries par la construction de nouveaux équipements destinés à régler les problèmes d'insalubrité et de surpeuplement. L'emprise de la prison étant insuffisante, elle fut complétée par l'emplacement de l'ancienne gendarmerie, cédé gratuitement à l'État par la Ville, ce qui porta la superficie disponible à 11000 mètres carrés.

Le contexte historique et patrimonial du terrain représenta un élément déterminant pour le projet architectural. La prison avait été édifiée sur les ruines du fort du Hâ dont étaient conservées deux tours et une portion de courtine qu'il n'était guère envisageable de faire disparaître d'autant qu'elles furent, à l'initiative de l'archiviste municipal de Bordeaux Jean-Paul Avisseau, inscrites à l'Inventaire supplémentaire des monuments historiques le 12 avril 1965, au cours de l'élaboration du projet. L'architecte fut donc contraint de les intégrer dans son plan et même d'en tirer parti comme nous le verrons plus loin. Au-delà de ces deux vestiges, ce fut surtout la proximité avec la cathédrale et l'hôtel de ville qui limita l'ampleur des futures constructions, sans pour autant interdire de leur donner une expression architecturale contemporaine.

Le terrain avait la forme d'un triangle rectangle dont la base, au sud, était bordée par le palais de justice et le côté perpendiculaire délimité à l'est par la rue du Maréchal-Joffre, c'est-à-dire peu dégagé et enclavé dans des constructions particulières en direction du nord et de l'ouest. L'élargissement de la rue des Frères-Bonie décidé en 1969 afin de désenclaver le quartier de l'hôtel de ville et d'y faire pénétrer la circulation automobile n'offrit pas pour autant une ouverture supplémentaire à cause des vestiges du fort du Hâ qu'il n'était définitivement plus possible de raser en raison de la protection dont ils bénéficiaient.

D'une manière symbolique, le futur établissement de formation des magistrats devait s'insérer dans un îlot urbain déjà en grande partie consacré aux fonctions judiciaires et pénitentiaires. Ce n'était pas un hasard, et cela avait même un sens très fort ${ }^{12}$.

\section{Le parti architectural : traduction moderne d'une architecture parlante et symbolique}

Dès sa nomination par la Chancellerie, Guillaume Gillet se consacra à l'étude du parti architectural. Le premier obstacle à affronter était celui de l'implantation des futurs bâtiments, compte tenu de toutes les contraintes citées précédemment. Au printemps 1961, il étudia plusieurs solutions allant du plan compact à la dispersion des différents éléments sur le terrain en passant par la mise à l'alignement sur les limites physiques [fig. 2]. 
Figure 2

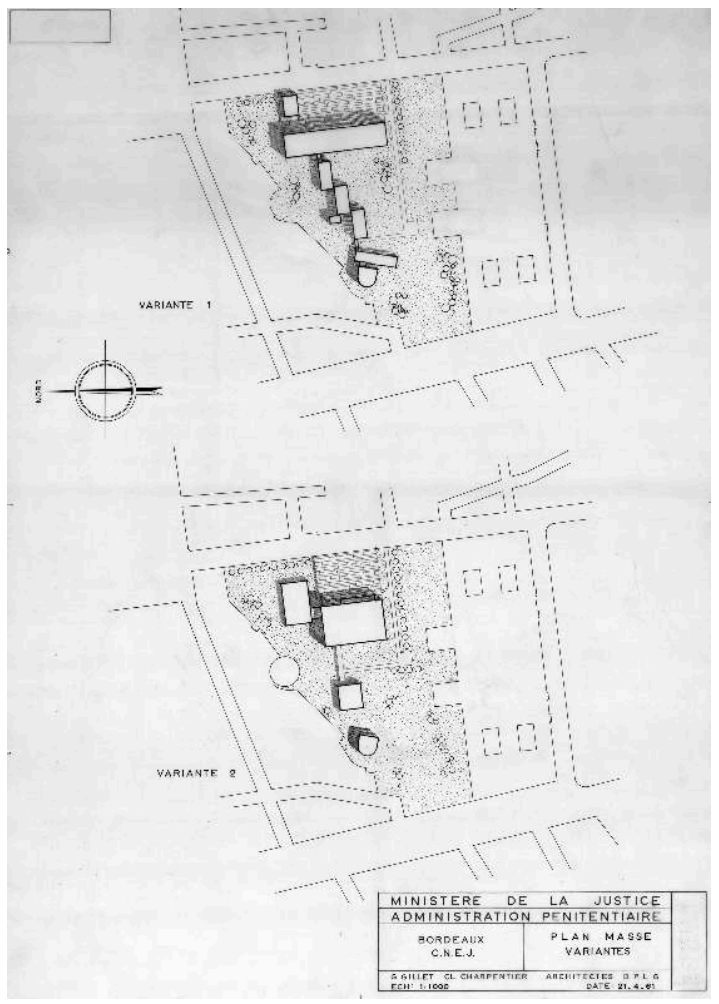

Études de plan de masse au 1/1000e, avril 1961, conservé au SIAF / Cité de l'architecture et du patrimoine, centre d'archives, fonds Guillaume Gillet.

(c) Guillaume Gillet / reproduction Franck Delorme.

La principale difficulté résidait dans la confrontation avec les tours médiévales, qu'il était possible de résoudre soit par la distanciation, soit par l'intégration, hésitation dont témoignent de nombreuses esquisses présentes dans les archives de l'architecte conservées à la Cité de l'architecture et du patrimoine. La seconde contrainte était l'enclavement du terrain dont seul le côté est ouvrait sur la rue du Maréchal-Joffre ; sur ses deux autres côtés, il était entouré de constructions. Une ouverture vers l'ouest, du côté du cours d'Albret, fut d'abord envisagée pour donner accès aux logements de fonction ; elle fut par la suite abandonnée.

Les recherches montrent tour à tour les éléments du programme répartis dans une grappe de petits volumes dans laquelle sont intégrées les deux tours, ou bien ces mêmes éléments rassemblés dans un seul bâtiment creusé par un large patio et dans lequel les tours sont englobées, jusqu'à disparaitre [fig. 3]. 
Figure 3

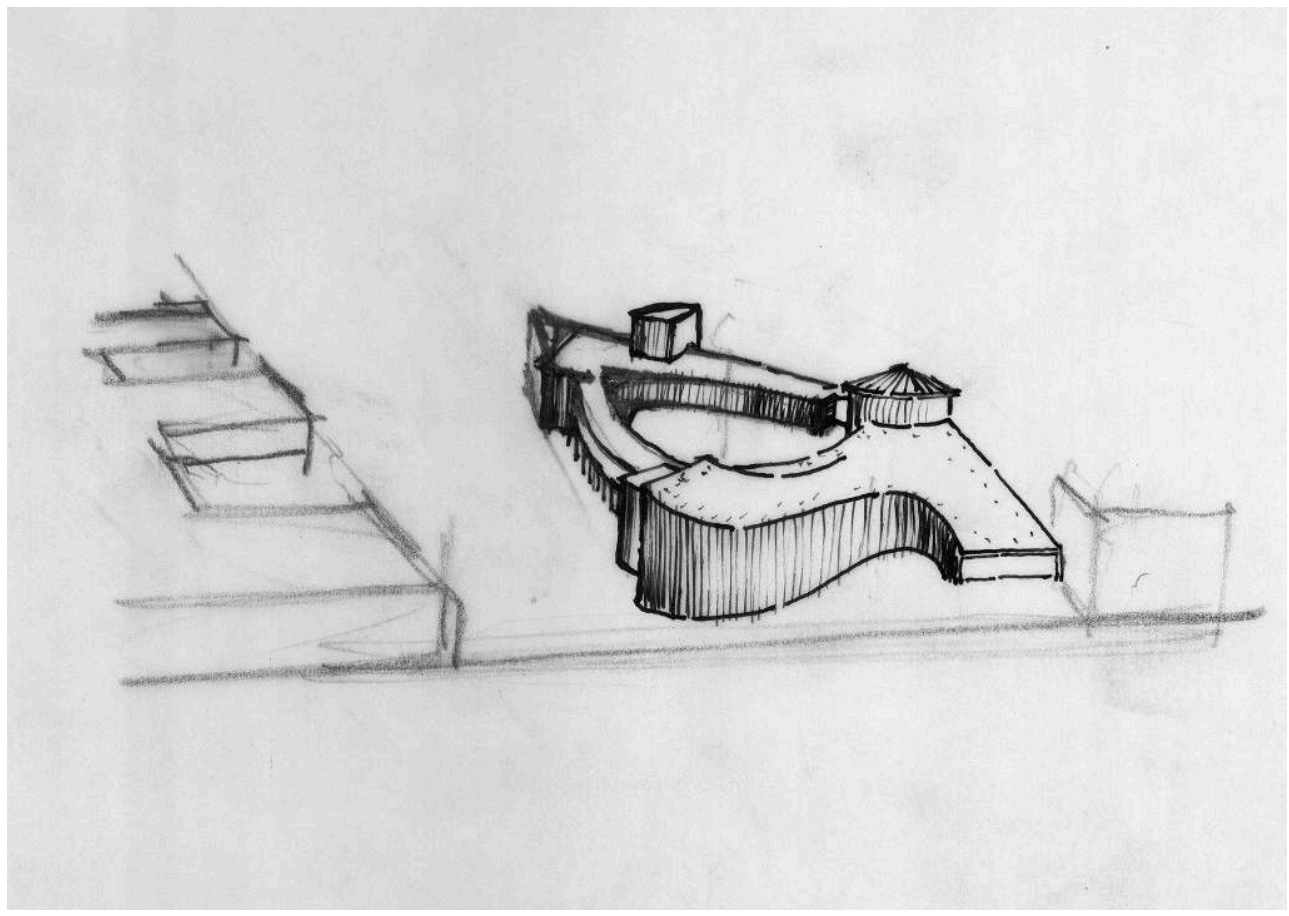

Croquis d'études, n.d, conservé au SIAF / Cité de l'architecture et du patrimoine, centre d'archives, fonds Guillaume Gillet.

(c) Guillaume Gillet / reproduction Franck Delorme.

Il semble qu'aucun de ces partis ne donna satisfaction à l'architecte car ils débouchaient sur une forme "molle » et assez indéterminée, sans doute très éloignée de ses idéaux de monumentalité et de régularité. Ce fut finalement un parti envisagé dès le mois de novembre 1960 qui fut retenu, celui d'un bâtiment disposé en équerre et dont les deux branches s'appuient sur les limites sud et est, dégageant le reste du terrain et les vestiges médiévaux [fig. 4]. À quelques variations près, ce parti d'implantation fut conservé jusqu'au bout et même accentué au fur et à mesure de la définition du projet. 
Figure 4

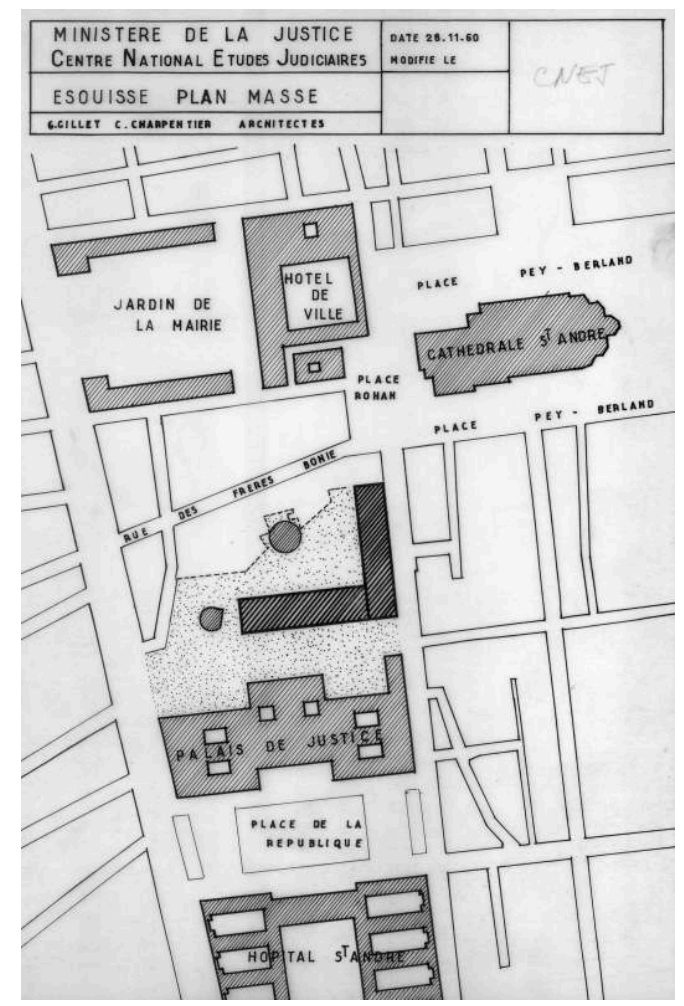

Principe d'implantation du Centre national d'études judiciaire au 1/1000', novembre 1960, conservé au SIAF / Cité de l'architecture et du patrimoine, centre d'archives, fonds Guillaume Gillet.

(c) Guillaume Gillet / reproduction Franck Delorme.

La principale difficulté restait le rapport physique que le futur bâtiment entretiendrait avec les deux tours de l'ancien fort. Dans un premier avant-projet, l'École prend la forme d'un bâtiment continu prenant appui sur l'ensemble des limites du terrain, sauf du côté de la rue du Maréchal-Joffre où la façade est en retrait.

Les tours sont incluses dans le périmètre des bâtiments mais sans que leurs volumes disparaissent dans l'ensemble. Une grande cour centrale quasi circulaire est dégagée. En contrepoint, l'entrée ouvre sur un hall de plan parabolique. Les élévations est et sud sont divisées chacune en douze travées fortement rythmées par une structure en béton armé visible en façade [fig. 5]. 
Figure 5

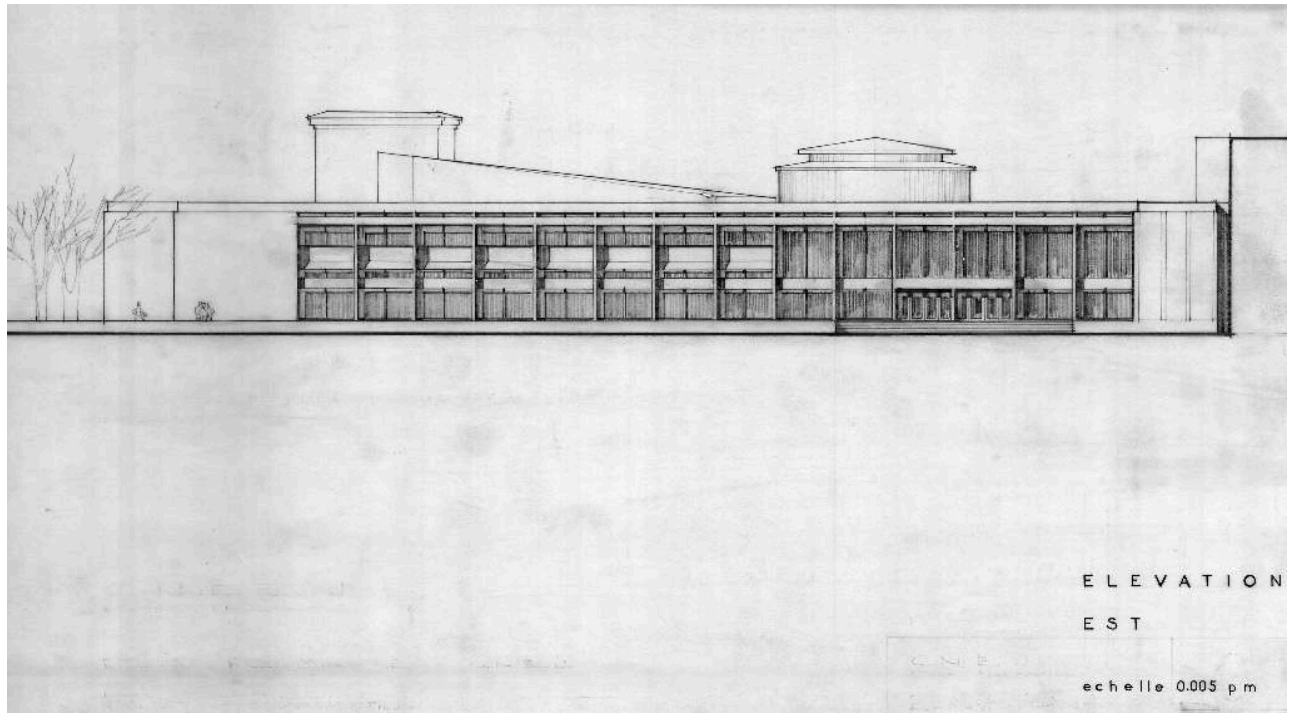

Étude de l'élévation est au $1 / 200^{e}$, n.d, conservé au SIAF / Cité de l'architecture et du patrimoine, centre d'archives, fonds Guillaume Gillet.

Reproduction Franck Delorme.

Dans un second avant-projet, la disposition d'ensemble est la même mais l'École ne dessine plus qu'une équerre dont l'angle rentrant est adouci au moyen d'une ample courbe dont l'extrémité nord a pour point de départ la grosse tour des Minimes alors qu'à l'autre extrémité, la tour des Anglais est détachée. Une contre-courbe infléchit la façade du bâtiment sur la rue pour accueillir un parvis d'entrée entre la salle de conférences et l'accueil. La bibliothèque, un des éléments principaux du programme, occupe l'autre aile, emplacement qui sera le sien dans la réalisation; initialement prévue sur deux niveaux, elle occupe finalement tout l'étage [fig. 6]. 


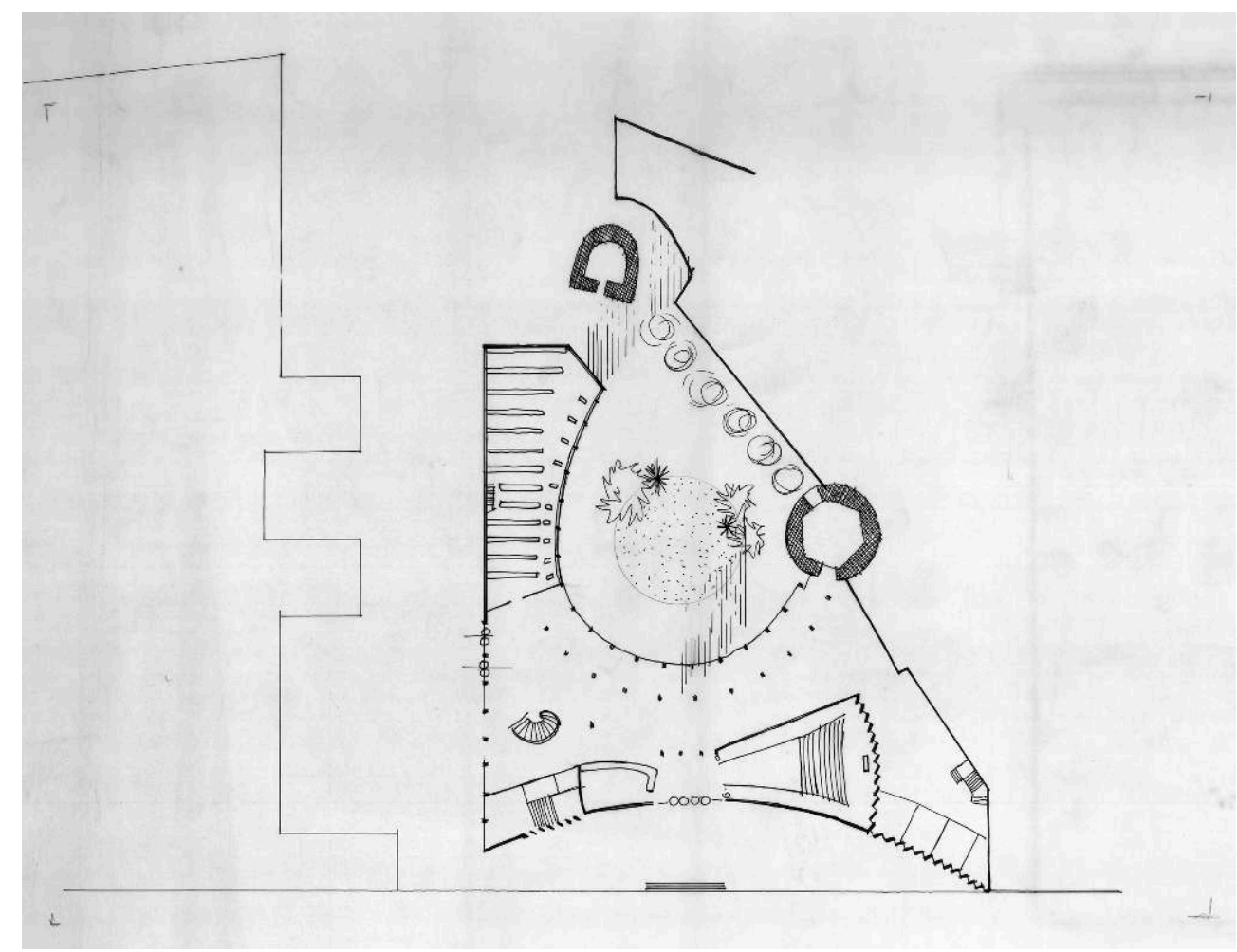

Étude du plan du rez-de-chaussée au $1 / 500^{\circ}$, n.d, conservé au SIAF / Cité de l'architecture et du patrimoine, centre d'archives, fonds Guillaume Gillet.

(c) Guillaume Gillet / reproduction Franck Delorme.

En mars 1962, le troisième et dernier avant-projet conserve ce parti d'ensemble et le renforce en donnant plus de régularité aux différents volumes. La partie administrative et la salle de conférences sont réunies dans un parallélépipède épais à l'est tandis que la bibliothèque et les salles de formation sont dans l'aile en retour, plus étroite. Cette répartition ne changera plus. Une vaste salle des pas perdus prend place dans le creux de l'équerre. Sa façade sur la cour dessine une parabole régulière dont le centre géométrique semble être la grosse tour des Minimes désormais complètement isolée car Gillet entrevoit bien que le meilleur moyen de garder aux vestiges du fort toute leur dimension patrimoniale est de maintenir son bâtiment à une distance respectueuse.

Cet avant-projet a fait l'objet d'une maquette [fig. 7] présentée au mois d'avril 1962 lors d'une réunion de travail chez le préfet Gabriel Delaunay, ce qui donne bien la mesure de la portée du projet, encore amplifié dans la presse régionale par le surnom de « Nouveau Saint-Cyr de la magistrature ${ }^{13}$ ». La mise au point de l'avant-projet débuta en juillet 1962. Il s'écoula ensuite deux années avant que le projet ne soit soumis à l'examen du Conseil des bâtiments de France, qui l'approuva tout en attirant l'attention de l'architecte sur l'aspect des futures façades ${ }^{14}$. Le projet prévoyait qu'elles soient constituées par de grands panneaux de béton séparés par des vides verticaux, solution proche de celle que Gillet avait choisie pour les élévations des maisons d'arrêt qu'il concevait au même moment à Valenciennes (Nord), Muret (Haute-Garonne) et surtout Bordeaux-Gradignan. 
Figure 7

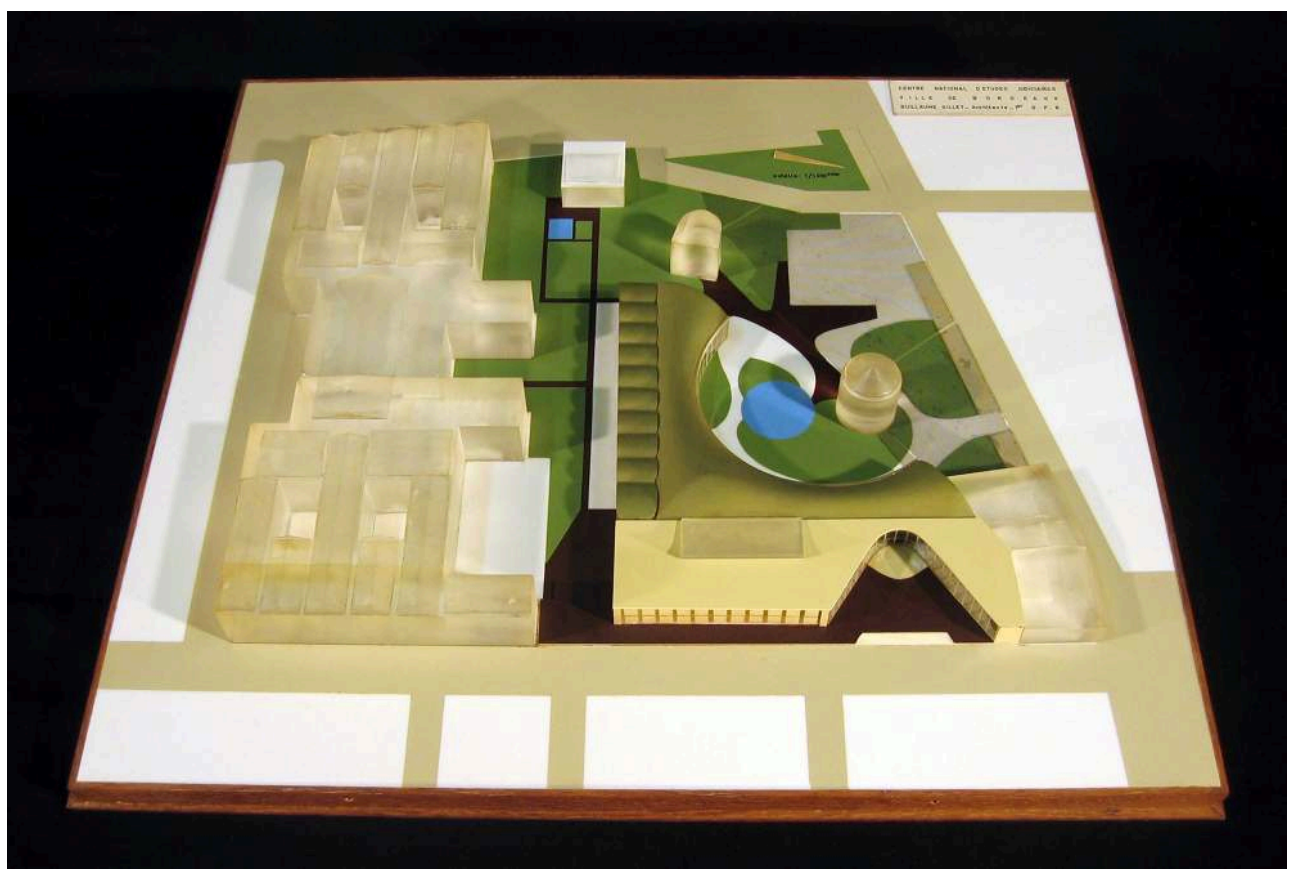

Maquette de l'avant-projet définitif au 1/500, n.d, conservée au SIAF / Cité de l'architecture et du patrimoine, centre d'archives, fonds Guillaume Gillet.

(c) Guillaume Gillet / reproduction Franck Delorme.

L'école tourne en quelque sorte le dos à la façade arrière du palais de justice, ce qui permet d'aménager entre les deux bâtiments une rue intérieure pour l'accès au parking souterrain de l'École, aux locaux de services, ainsi qu'au parking des véhicules de police au palais de justice. Un volume carré renfermant les logements de fonction occupe le fond du terrain, du côté du cours d'Albret où il a son entrée indépendante.

\section{Une construction très rapide}

De 1964 à 1969, le projet a subi une longue interruption en raison de la lenteur prise par la libération des terrains et de l'incertitude concernant le financement de la construction. Les études ne reprirent qu'au premier trimestre de l'année 1967, au moment de l'achèvement et de la mise en service de la nouvelle maison d'arrêt à Gradignan. Le transfert des prisonniers permit l'évacuation des locaux et leur destruction.

En 1969, à partir des observations et de la prise en compte de nouvelles données programmatiques, la partie du bâtiment réservée à l'administration et à la salle de conférences fut modifiée. Elle prit l'aspect d'une barre épaisse et parallèle à la rue du Maréchal-Joffre dont la partie centrale renferme la salle de conférences. L'autre aile, de moindre épaisseur, est destinée aux locaux d'enseignement proprement dits [fig. 8]. Des patios permettent d'éclairer les locaux éloignés des façades. 
Figure 8

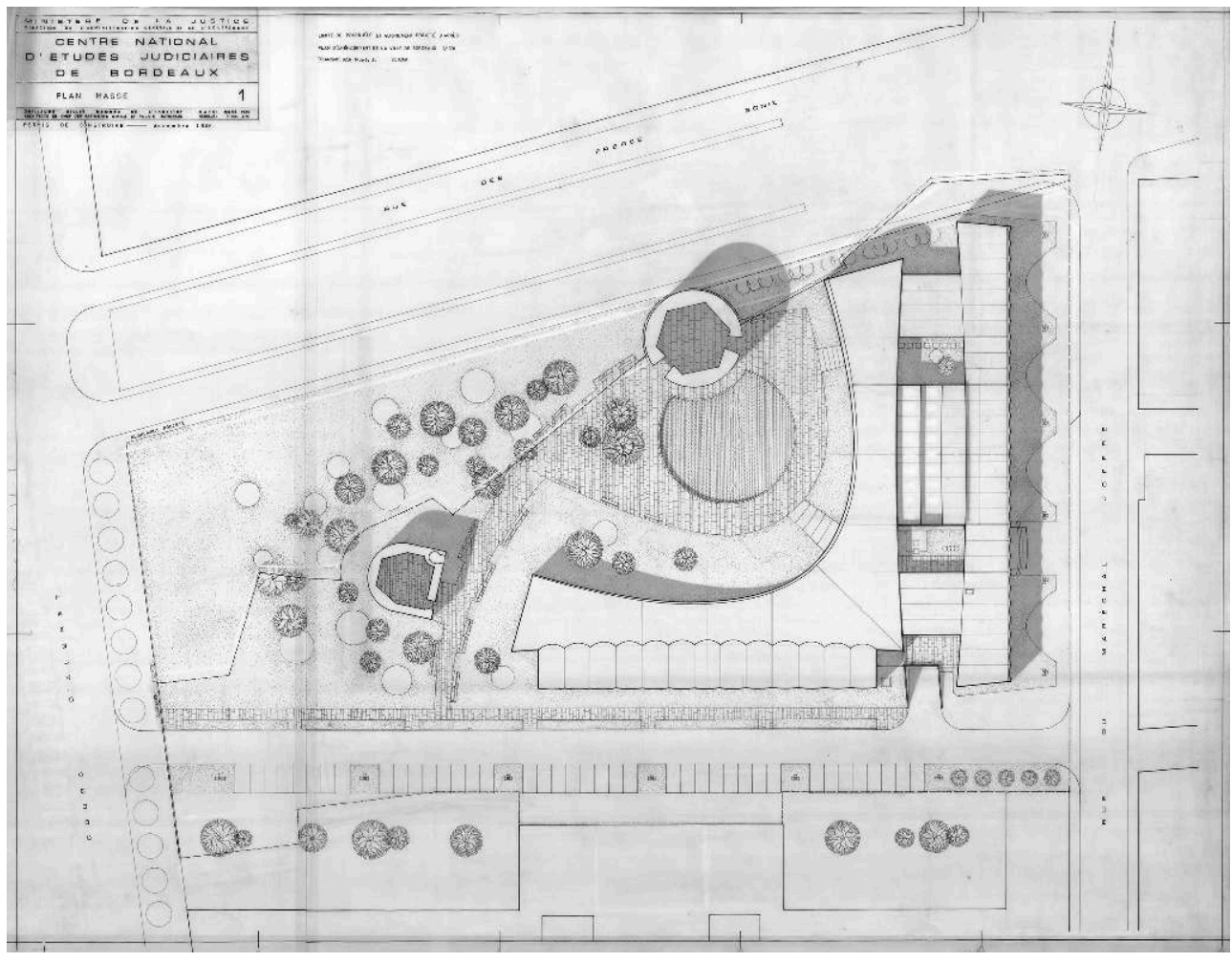

Plan de masse au $1 / 200$ e présenté pour le permis de construire, décembre 1969, conservé au SIAF / Cité de l'architecture et du patrimoine, centre d'archives, fonds Guillaume Gillet.

(c) Guillaume Gillet / reproduction Franck Delorme.

Les élévations sont prévues pour être largement vitrées et fortement rythmées par des grands éléments verticaux espacés régulièrement et entre lesquels un système de brisesoleil semble être envisagé. Seule la façade face au palais de justice n'est pas traitée de manière uniforme en raison de son exposition au sud et du fait de la présence à l'étage des magasins de la bibliothèque. En revanche, au rez-de-chaussée, les salles de réunion sont plus généreusement éclairées. Les pignons sont déjà prévus pour être recouverts de dalles de pierre pour faire écho aux murs massifs des tours [fig. 9]. La demande de permis de construire est déposée en décembre 1969.

Figure 9

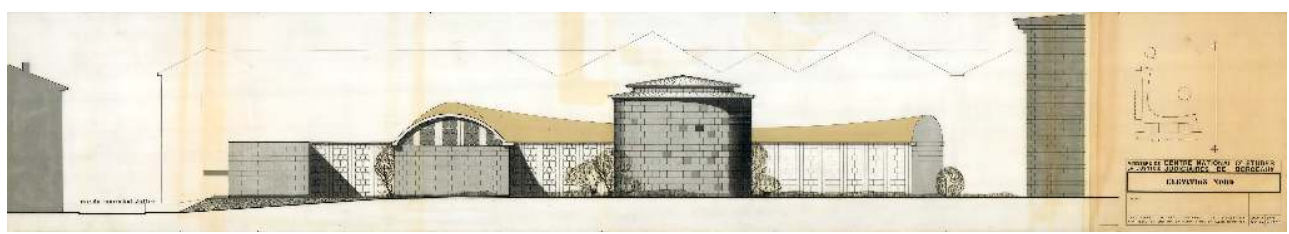

Élévation nord au 1/200e, avril 1970, conservé au SIAF / Cité de l'architecture et du patrimoine, centre d'archives, fonds Guillaume Gillet.

(c) Guillaume Gillet / reproduction Franck Delorme.

Le directeur de l'administration centrale du ministère de la Justice ayant demandé le 5 janvier 1963 à Guillaume Gillet de désigner un confrère résidant à Bordeaux pour remplir la fonction d'architecte d'opération chargé de veiller à la bonne marche des procédures et des travaux, il choisit Paul Daurel (1920-?). Celui-ci avait de nombreux 
chantiers à son actif, dans le domaine universitaire notamment, en collaboration avec les architectes Louis Sainsaulieu, Pierre Mathieu et Claude Bouey, mais aussi avec l'architecte américain Marcel Breuer pour les laboratoires pharmaceutiques Sarget (Mérignac, 1967). À Paris, Gillet était secondé par Bernard Cayla (?-1994), un de ses deux chefs d'agence, qui avait en charge tous les projets pour le ministère de la Justice. Ayant pour habitude de recourir aux ingénieurs, Gillet fit appel à Robert Lourdin, spécialiste des charpentes en bois lamellé-collé, avec lequel il avait déjà travaillé pour les églises de Vieux-Condé (Nord; Notre-Dame-de-la-Solitude, 1961-1966), Avignon (Vaucluse ; Saint-Joseph-Travailleur, 1967-1969) et Châtenay-Malabry (Hauts-de-Seine, chapelle du collège du Sacré-Cœur, 1964-196915).

Après destruction des bâtiments de l'ancienne prison, les travaux de construction de l'ENM débutèrent au printemps 1971 et furent conduits avec une rapidité étonnante en comparaison du nombre d'années qu'il fallut pour parvenir au projet définitif. En mars 1972, le gros œuvre de béton armé des deux ailes fut achevé par l'entreprise Chassinat de Paris. En juillet, ce fut au tour des piliers et de la corniche de la salle des pas perdus d'être coulés. La charpente conçue par Robert Lourdin [fig. 10] est constituée d'une série d'arcs surbaissés qui ont la même courbe mais des écartements différents en fonction de la largeur variable existant entre les parois des ailes auxquelles est adossée la salle des pas perdus et la paroi vitrée courbe qui la ferme du côté de la cour. À l'extérieur, cela confère à la toiture l'allure d'un dos de bête gigantesque qui ondoie; ce mouvement tempère la sévérité qu'aurait pu avoir le bâtiment.

Figure 10

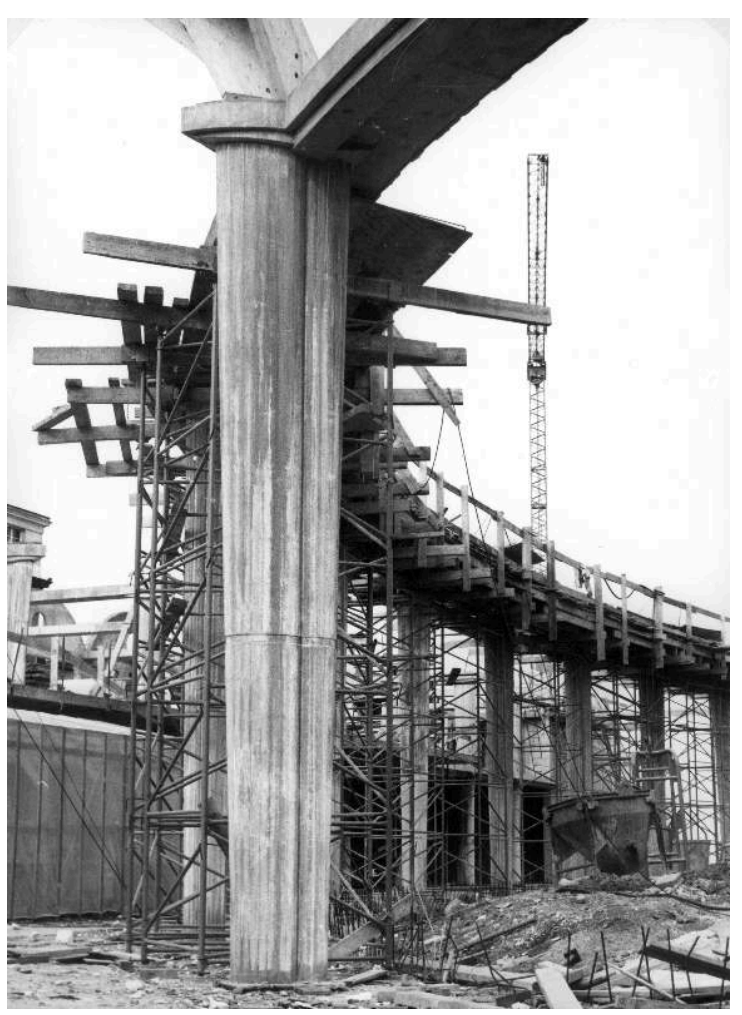

Poteau et corniche en béton armé de la salle des pas perdus en cours de chantier, n.d, conservé au SIAF / Cité de l'architecture et du patrimoine, centre d'archives, fonds Guillaume Gillet.

(C) Burdin / (c) Guillaume Gillet / reproduction Franck Delorme. 
L'aménagement intérieur fut mené à bien entre septembre 1972 et le début de l'année 1973. Entretemps, l'ENM fut officiellement inaugurée le 12 décembre 1972 en présence du garde des Sceaux René Pleven, du maire de Bordeaux Jacques ChabanDelmas, de Maurice Aydalot (1904-1996), premier président de la Cour de cassation et président du conseil d'administration de l'établissement.

\section{L'ENM, une architecture de style « Ve République » ?}

Les Trente Glorieuses ont été marquées par un niveau d'équipement sans précédent. La plupart d'entre eux, telle l'aérogare internationale d'Orly inaugurée par Charles de Gaulle en 1961, bien que le projet remonte à 1957, c'est-à-dire avant son retour au pouvoir, symbolisent cette période de prospérité et de réorganisation administrative du pays. Ils sont de divers ordres et appartiennent à différents domaines : politique, économique, social ou culturel. Au même titre que certains de ses confrères, Prix de Rome ou BCPN comme lui, Gillet a participé à plusieurs reprises à cet équipement architectural. Le programme immobilier mené pour l'administration pénitentiaire, déjà cité, en fait partie, mais aussi le palais des congrès de la porte Maillot à Paris (1966-1985) ou l'aéroport de Lyon-Satolas (1969-1986), montrant à quel point l'équipement économique fut aussi important que le politique.

41 En réponse au critique André Fermigier qui qualifiait le palais des congrès de "bâtiment honnête digne d'un Grand Prix de Rome », Gillet avoua ne pas être opposé à la définition d'un «style V ${ }^{e}$ République » car il défendait le droit et avant tout le devoir d'un architecte de produire une architecture contemporaine utilisant le langage et les matériaux modernes pour « marquer son époque ${ }^{16}$ ».

À l'ENM, l'utilisation de matériaux nouveaux et contemporains n'exclut pas la beauté de quelques «gestes » et le traitement «noble » de certains éléments. Les façades sont revêtues de panneaux et de brise-soleil en aluminium anodisé. Les pignons sont revêtus de pierre calcaire blanche. Une frise d'hexagones aplatis ceinture tout l'édifice, donnant une unité à l'ensemble des façades [fig. 11]. 
Figure 11

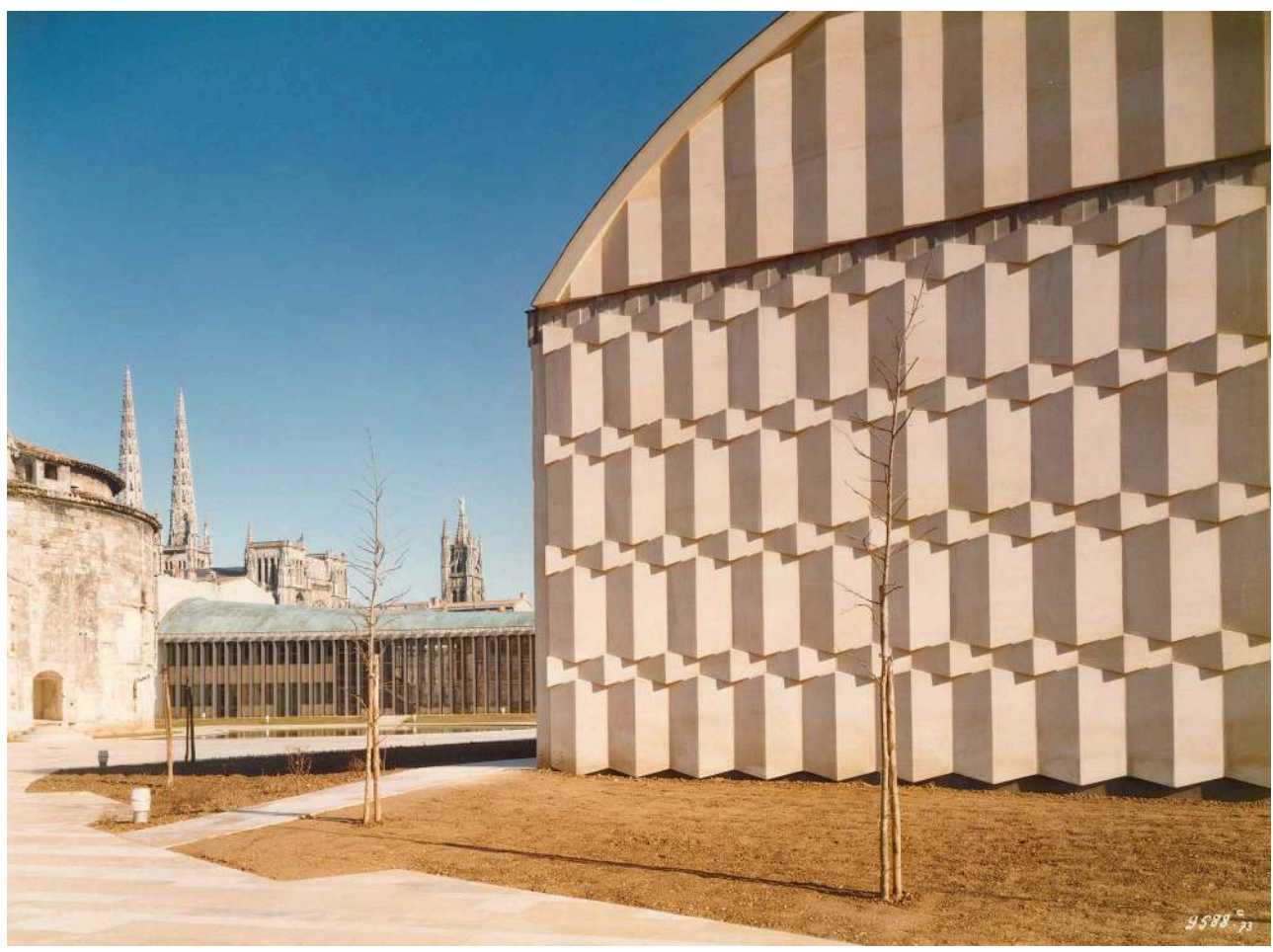

Cour de l'ENM avec au premier plan le pignon ouest de la salle des pas perdus, n.d., photographie conservée au SIAF / Cité de l'architecture et du patrimoine, centre d'archives, fonds Guillaume Gillet.

(c) ISO Bordeaux / (c Guillaume Gillet / reproduction Franck Delorme.

Gillet a transposé dans une certaine modernité, au moins des matériaux, un vocabulaire monumental traditionnel. Les piliers en béton armé de la salle des pas perdus, de section en double ovale, par leurs facettes et leur évasement vers le haut [fig. 12], outre qu'ils évoquent les colonnades à l'antique des palais de justice du xixe siècle, puisent aussi dans l'architecture d'Auguste Perret (1874-1954), un des maitres de Gillet, à travers les exemples du Mobilier national (1936) ou du musée des Travaux publics (1937, aujourd'hui palais d'Iéna, siège du Conseil économique, social et environnemental) à Paris. 
Figure 12

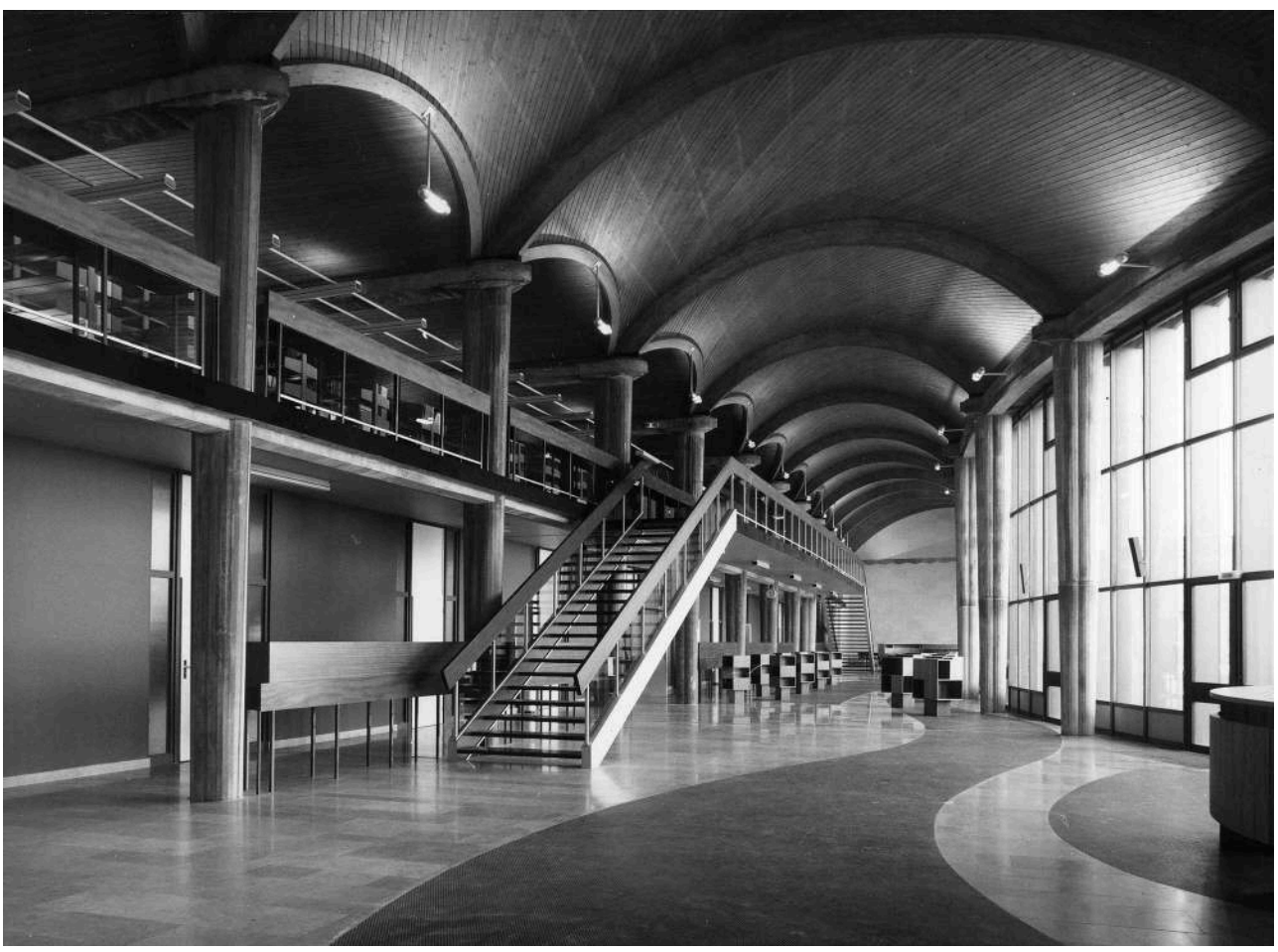

Salle des pas perdus du côté de la bibliothèque, n.d, photographie conservée au SIAF / Cité de l'architecture et du patrimoine, centre d'archives, fonds Guillaume Gillet.

(c) Burdin / (c) Guillaume Gillet / reproduction Franck Delorme.

\section{Aménagement intérieur}

Espace majeur de l'école, la salle des pas perdus permet la liaison entre les différentes parties du programme; elle est aussi un lieu de rencontres, d'échanges, de débats, à l'instar de la salle des pas perdus d'un palais de justice. Dans sa forme de demi-cloître, elle est l'espace de déambulation et de discussion de l'«académie socratique » définie par Gillet. La protection dont elle bénéficie lui donne «cette situation dominante qui met la Justice au-dessus de l'agitation immédiate de la rue ${ }^{17}$ ».

Baignée par la lumière du nord-ouest qui entre généreusement par la façade vitrée de la cour, elle revêt à plusieurs égards un caractère symbolique. La succession d'éléments verticaux qui la clôt du côté de la cour évoque peut-être la haie de branchages que, jadis en Occident, on dressait à l'extérieur des villes pour délimiter le lieu de justice. Elle entoure et embrasse la tour des Minimes qui devient le point de mire, tenant la même position que le juge dans un tribunal, qui occupe, selon Robert Badinter « une position centrale autour de laquelle tout s'ordonne, comme le débat judiciaire qu'il conduit $^{18}{ }$. La tour est ainsi le centre à la fois physique et symbolique de l'ENM.

L'aménagement et le traitement de la salle des pas perdus ont été pensés et réalisés avec soin, tant par le choix des matériaux, du mobilier et des interventions artistiques. Le sol est recouvert d'un dallage de Comblanchien et de tapis de pastilles de céramique noire, le tout dessinant « des courbes qui se déploient, se développent et progressent telles les méandres d'un fleuve incitant à un cheminement et à une découverte poétique ». Le mur qui sépare le hall de la salle de conférences est recouvert d'une 
composition peinte en grands aplats de deux teintes, rouge et brun, sur un fond ocre [fig. 13], œuvre de Monique Poncelet (1931-), professeur à l'École des beaux-arts de Paris. C'est une « allégorie sur le thème de la vie et de l'espoir » faite de «formes aux tonalités sombres situées à la gauche de la composition et laissent progressivement place, vers la droite à des surfaces qui s'allègent, s'élèvent et se terminent, au-delà de l'entrée, dans des valeurs lumineuses qui éclatent telles des gerbes de générosité et d'espérance ${ }^{19} »$.

Figure 13

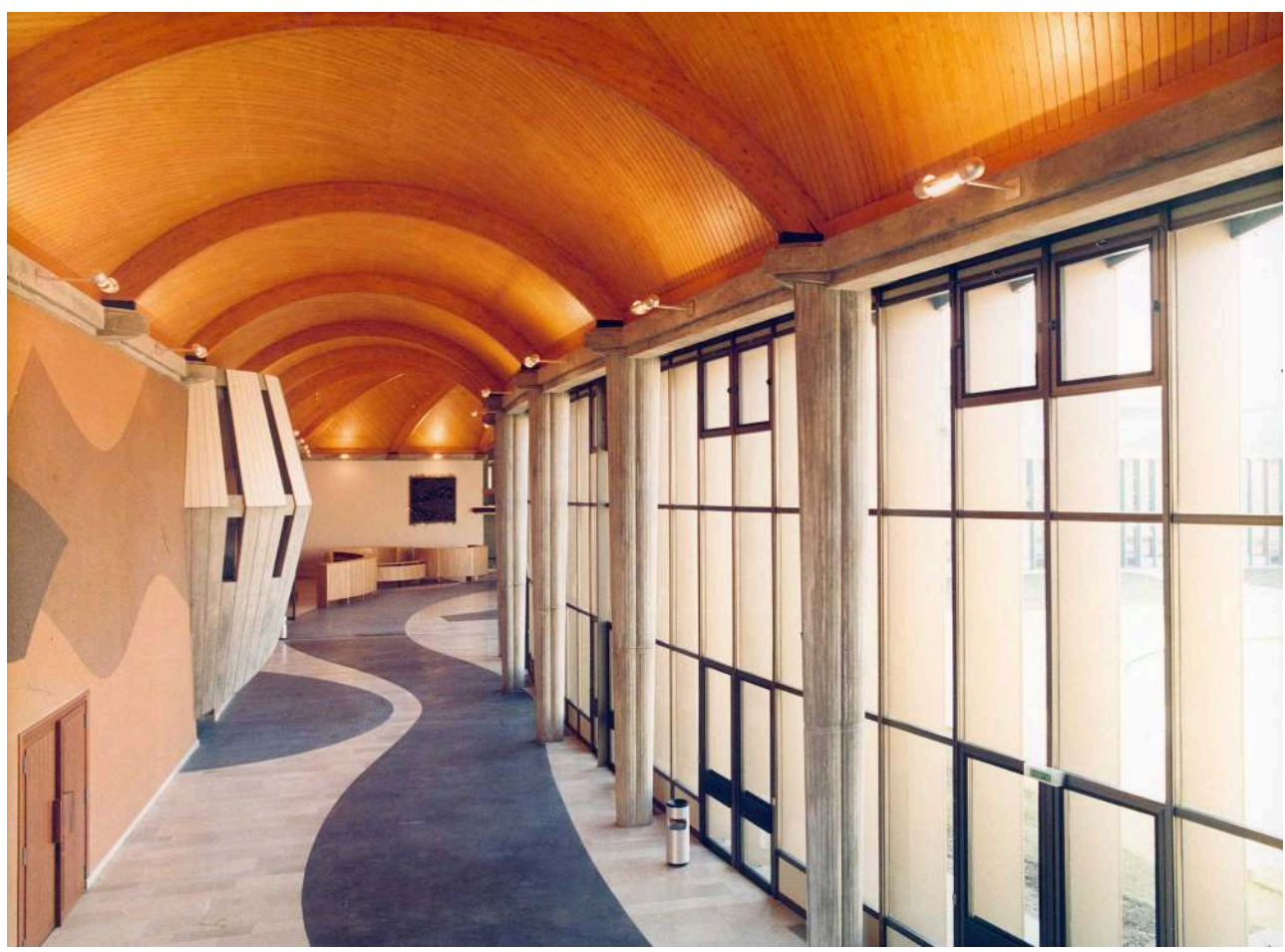

Composition peinte de Monique Poncelet dans la salle des pas perdus, photographie conservée au SIAF / Cité de l'architecture et du patrimoine, centre d'archives, fonds Guillaume Gillet.

(C) Burdin / (c) Guillaume Gillet / reproduction Franck Delorme.

47 La cabine de projection, sorte de concrétion de béton armé suspendue au mur de la salle de conférences, est constituée de deux troncs de cônes soudés par leur base. Celui du bas est découpé par la spirale de l'escalier qui la rattache au sol ; celui du haut est percé de meurtrières. L'aspect général fait penser à une chaire de lecteur dans le réfectoire d'un couvent ; c'est d'ailleurs ainsi qu'on la surnomme à l'ENM [fig. 14]. 
Figure 14

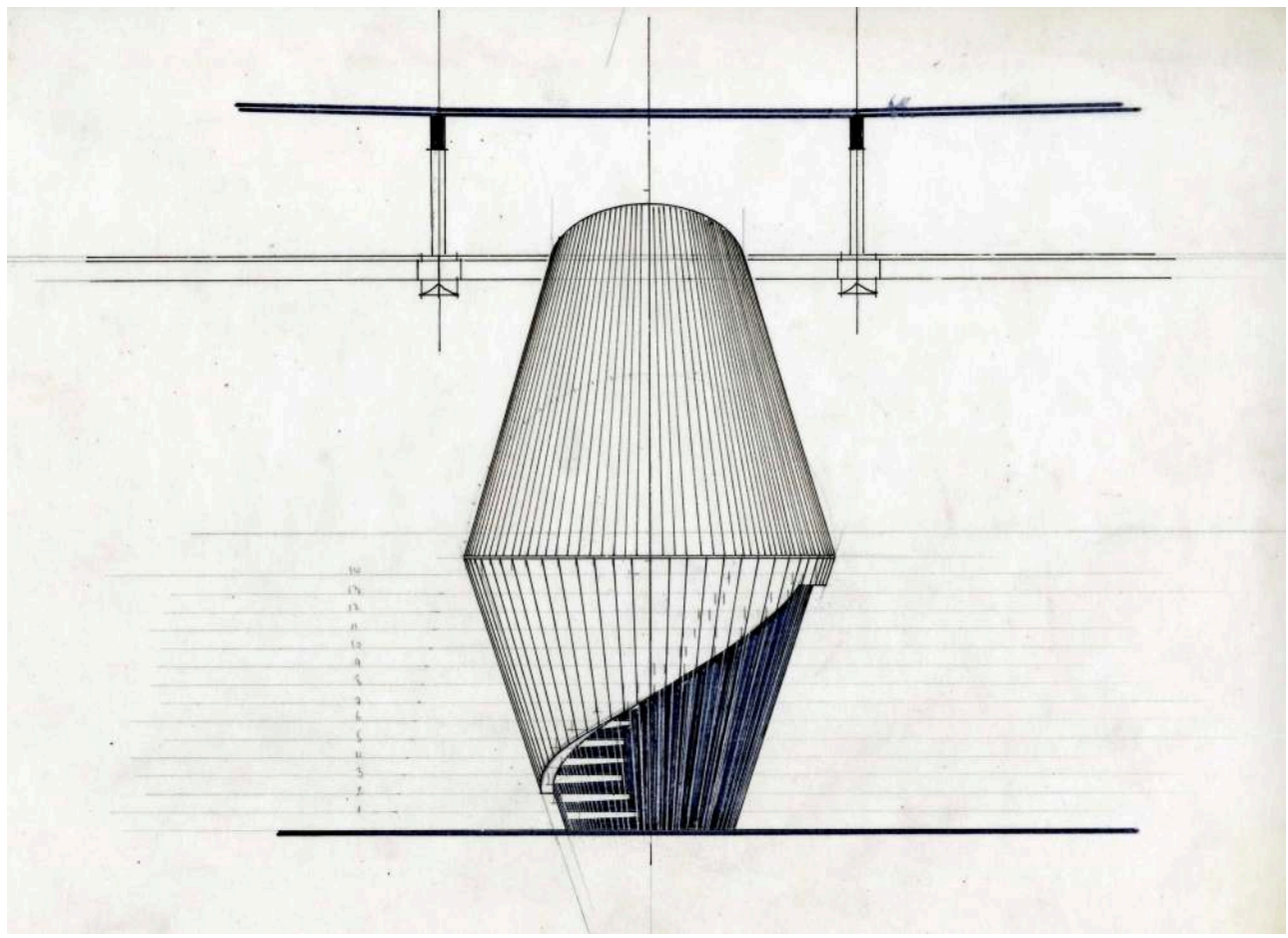

Élévation de la cabine technique de la salle de conférences, n.d, dessin conservé au SIAF / Cité de l'architecture et du patrimoine, centre d'archives, fonds Guillaume Gillet.

(c) Guillaume Gillet / reproduction Franck Delorme.

Après avoir dans un premier temps envisagé de faire appel à l'Atelier de recherche et de création (ARC) du Mobilier national ${ }^{20}$, Gillet s'est finalement décidé à concevoir luimême les éléments qui meublent la salle des pas perdus, à commencer par la banque d'accueil, aujourd'hui disparue, qui se trouvait à l'intersection des deux ailes, face à l'ancien accès.

L'espace de lecture de la bibliothèque est fait d'un ensemble d'alvéoles circulaires dont les dossiers des banquettes reçoivent des rayonnages, complétés par de petits casiers cubiques. Dessiné par l'architecte et son agence, ce mobilier a été fabriqué par la Manufacture parisienne de sièges qui réalisa aussi les gradins de l'auditorium, remplacés dans les années 1990 [fig. 15]. Dans l'extrémité nord du hall, l'ancienne cafétéria était meublée de sièges et de tables en plastique moulé bien caractéristiques du mobilier des années 1970. 


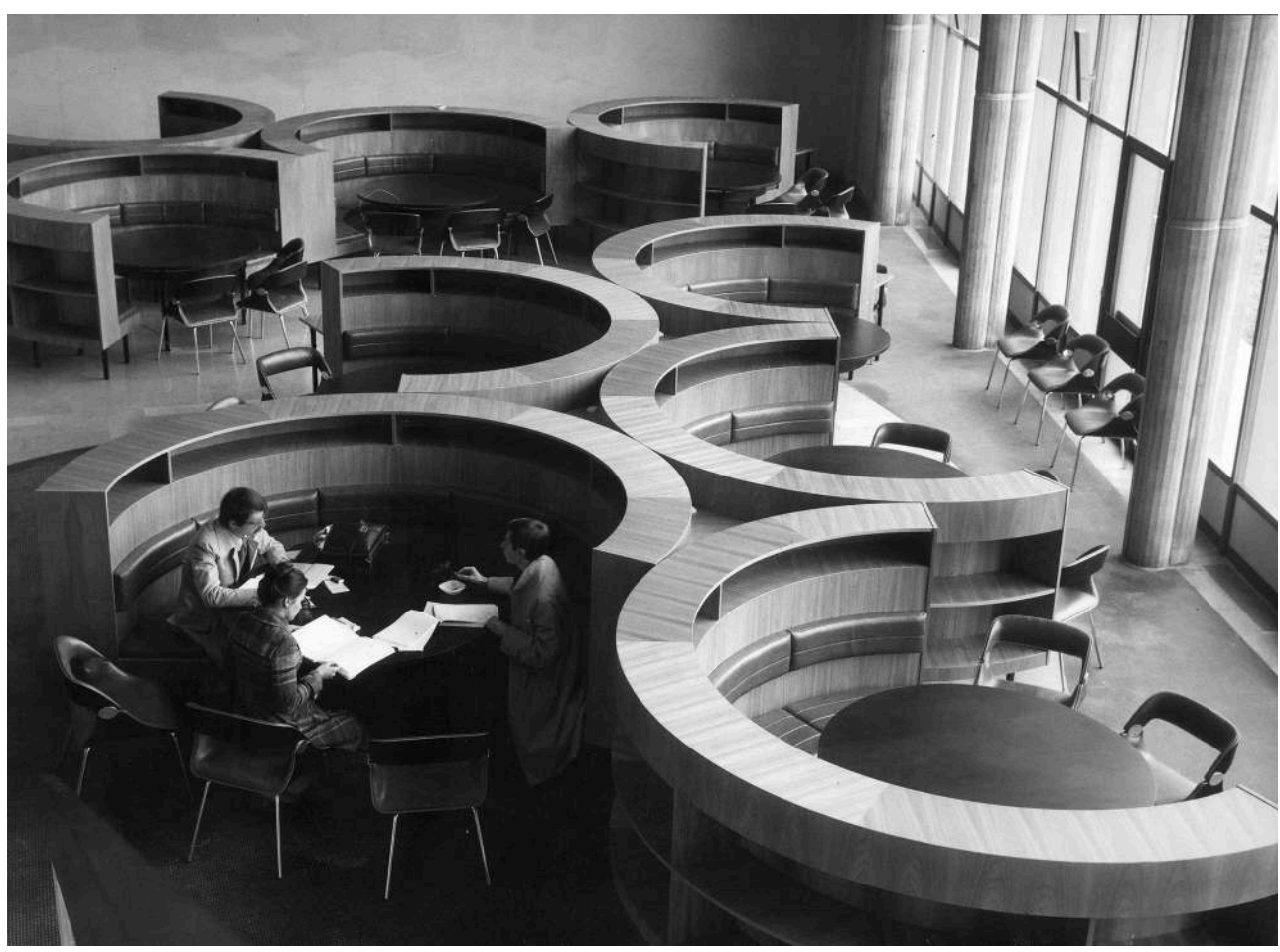

Banquettes et tables de lecture conçues par Gillet et réalisées par la Manufacture parisienne de sièges, n.d., photographie conservée au SIAF / Cité de l'architecture et du patrimoine, centre d'archives, fonds Guillaume Gillet.

(c) Burdin / (c) Guillaume Gillet / reproduction Franck Delorme.

\section{Les modifications ultérieures}

Depuis son ouverture, l'ENM a subi des transformations nécessitées par l'évolution de son fonctionnement et des besoins, dont les plus importantes ont été réalisées au moment de la construction du tribunal de grande instance (TGI). En 1987, lors du premier concours pour l'édification du TGI, remporté par le bordelais Jacques Hondelatte (1942-2002), il fut décidé de réaliser une extension et un réaménagement de l'ENM. En 1992, au second concours, le lauréat, l'anglais Richard Rogers (1933-), proposa deux extensions prenant chacune place à une des extrémités des ailes de l'école. La plus proche du futur TGI constitue une circulation entre les deux bâtiments et abrite une cafétéria commune au palais de justice, au TGI et à l'ENM. Le volume de la salle des pas perdus est prolongé par une sorte de serre dont l'aspect est loin d'avoir la même qualité que celui du bâtiment de Gillet. La tour des Anglais, épargnée lors de la construction de l'ENM, a donc été littéralement transpercée et phagocytée par un escalier, un ascenseur et un monte-charge; elle semble maintenant écrasée par ses deux voisins.

51 La modification la plus radicale a été effectuée à l'angle de la rue des Frères-Bonie, de la rue du Maréchal-Joffre et de la place Pey-Berland où une seconde extension a été ajoutée. Si elle a le mérite de resserrer le débouché de la rue des Frères-Bonie assez mal délimitée depuis la destruction des maisons adossées aux vestiges du fort du Hâ, cette construction est encore plus sujette à critique par sa forme de tour carrée et massive. C'est le résultat bâtard du bras de fer engagé entre le ministère de la Justice (par 
l'intermédiaire de la Direction immobilière), l'architecte et les services des Monuments historiques, ceux-ci ayant imposé des façades partiellement en pierre (plaquée) et une toiture en tuiles, en raison de la proximité de la cathédrale. Ce qui trente ans auparavant n'avait pas empêché Gillet de faire un bâtiment résolument contemporain est devenu un obstacle à l'innovation architecturale : autres temps, autres mœurs !

L'entrée qui se trouvait rue du Maréchal-Joffre a été condamnée, faisant ainsi disparaître la belle grille de serrurerie [fig. 16].

Figure 16

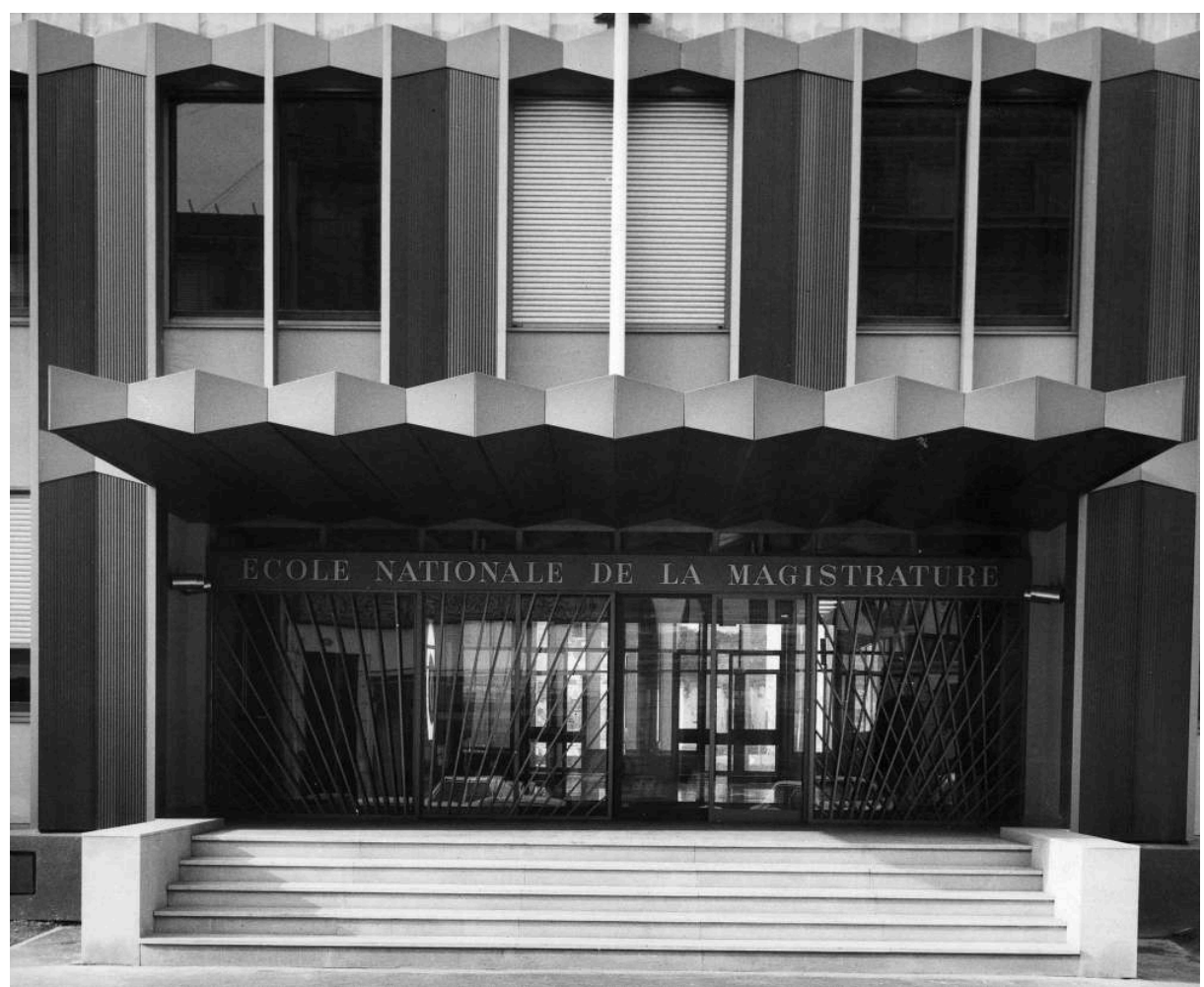

L'ancienne entrée de l'ENM rue du Maréchal-Joffre, photographie conservée au SIAF / Cité de l'architecture et du patrimoine, centre d'archives, fonds Gillet.

(c) Burdin / (c) Guillaume Gillet / reproduction Franck Delorme. pignon nord de la salle des pas perdus, ce qui représente un dévoiement total du fonctionnement et de la raison d'être du plan en équerre voulu par Gillet. Auparavant, le visiteur pénétrait au cœur du hall, face à la grande paroi vitrée découvrant l'ensemble de la salle des pas perdus et de ses deux ailes. Aujourd'hui, en pénétrant par une extrémité, le visiteur ne perçoit plus l'espace dans son ensemble et ne comprend ainsi plus la raison d'être de cette double galerie de cloître. transformation d'une grande partie de la bibliothèque-documentation en bureaux. $\mathrm{Si}$, récemment, le dallage de la cour a été entièrement refait à l'identique et le bassin remis en eau, en revanche, la substitution du vitrage de la salle des pas perdus par un vitrage plus isolant a entraîné la pose de barlotières dont le rythme horizontal entre en conflit avec le rythme vertical des brise-soleil et des piliers de cette même salle. Ces 
interventions ont fait perdre petit à petit au bâtiment de Gillet sa qualité et sa cohérence, voire son essence même.

L'ENM a bénéficié en $2007 \mathrm{du}$ label « Patrimoine du $\mathrm{Xx}^{\mathrm{e}}$ siècle », ce qui révèle, pour un bâtiment d'une période souvent décriée en architecture, son intérêt et sa parfaite insertion dans un environnement aux origines bien plus anciennes.

\section{NOTES}

1. Propos recueillis en 1987 lors d'un entretien accordé par Michel Debré.

2. Ordonnance $n^{\circ} 58-1270$, promulguée au Journal officiel du 22 décembre 1958. Voir https:// www.legifrance.gouv.fr/download/securePrint?token=kpVjdQ9vf93o6RyTMd@B [lien valide en octobre 2021].

3. Ordonnance $\mathrm{n}^{\circ}$ 59-77 du 7 janvier 1959.

4. Missions énoncées dans la loi organique portant statut de la magistrature (ordonnance $\mathrm{n}^{\circ}$ 58-1270 du 22 décembre 1958).

5. BOIGEOL Anne. «La formation des magistrats. De l'apprentissage sur le tas à l'école professionnelle ", Actes de la recherche en sciences sociales, Paris, Maison des sciences de l'homme, vol.76-77, 1989, p. 49-64, disponible en ligne https://www.persee.fr/doc/ arss_0335-5322_1989_num_76_1_2879 [lien valide en octobre 2021].

6. Discours d'inauguration de l'ENM par René Pleven en décembre 1972 retranscrit dans École nationale de la magistrature, Bordeaux, Delmas, 1972, p. 31.

7. BALMARY Jean, «Le Centre national d'études judiciaires », Le Pouvoir judiciaire, octobre 1969, p. 3.

8. , Note de présentation de Guillaume Gillet le 30 novembre 1972 reprise dans École nationale de la magistrature, p. 38.

9. DELORME Franck, «Guillaume Gillet, architecte: acteur majeur du renouveau du parc pénitentiaire dans les années 1960 », in M’BANZOULOU Pau \& DIEU François (dir.), L'Architecture carcérale. Des mots et des murs, Toulouse, Privat, coll. « Société et actu », 2012.

10. DELORME Franck, L'Église Notre-Dame de Royan. Guillaume Gillet et le gothique moderne, Bordeaux, Le Festin, 2012.

11. DELORME Franck, Guillaume Gillet, Paris, éditions du Patrimoine, coll. «Carnets d'architectes ", 2013.

12. DELORME Franck (dir.), Juger au cœur de la cité. L'îlot judiciaire bordelais, histoire et architecture, $d u \mathrm{XV}^{e}$ siècle à nos jours, Bordeaux, Confluences / École nationale de la magistrature, 2009.

13. Journal Sud-Ouest, 10 avril 1962, p. 5.

14. Ministère des Affaires culturelles, direction de l'Architecture, Conseil général des bâtiments de France, section spéciale des Bâtiments civils, séance du 10 décembre 1964, rapport dactylographié.

15. DELORME Franck, L'Église Notre-Dame de Royan. Guillaume Gillet et le gothique moderne.

16. GILLET Guillaume, «Le style V $V^{\mathrm{e}}$ République en architecture », Le Monde, 14 mars 1974, p. 25, disponible en ligne (pour les abonnés) https://www.lemonde.fr/archives/article/1974/03/14/lestyle-ve-republique-en-architecture_2514078_1819218.html [lien valide en octobre 2021].

17. BADINTER Robert (préface), La Justice en ses temples. Regards sur l'architecture judiciaire en France, Paris / Poitiers, Errance / Brissaud, 1992. 
18. BADINTER Robert (préface), La Justice en ses temples. Regards sur l'architecture judiciaire en France.

19. PONCELET Monique, "Description du programme de décoration du hall des pas perdus", document dactylographié, non daté, fonds d'archives Gillet, Cité de l'architecture et du patrimoine.

20. Voir la contribution de ZUBER-CUPISSOL Myriam in L'Atelier de Recherche et de Création du Mobilier national (1964-2000). Plaidoyer pour le mobilier contemporain. Hommage à Jean Coural, catalogue d'exposition (Galerie nationale de la Tapisserie, Beauvais, 15 mars-15 septembre 2001), Paris, Mobilier national, 2001, voir exemple p. 69.

\section{RÉSUMÉS}

Nommé en 1959 conseiller du ministère de la Justice, dont il a été en réalité le principal architecte pendant quinze ans, Guillaume Gillet (1912-1987) a été appelé à mener de front deux des plus importants programmes voulus par le garde des Sceaux Michel Debré : la construction de nouvelles prisons et la création d'une grande école des magistrats. Gillet est l'auteur d'une dizaine de lieux de détention qui renouvellent la conception de la prison, dans son aspect le plus fonctionnel comme dans son esthétique, reprenant ainsi le principe de l'architecture parlante et rationnelle.

En 1960, à Bordeaux (Gironde), pour l'École nationale de la magistrature, Gillet fut chargé de concevoir ce qui devait désormais être le lieu unique de formation de tous les magistrats de France, nouvelle institution prévue par la réforme judiciaire adossée à la Constitution de 1958 . Cette nouvelle fonction, l'architecte eut pour mission de l'incarner par des bâtiments contribuant à donner une image moderne du pouvoir judiciaire. Pour autant, l'architecte ne pouvait faire abstraction des caractères les plus marquants des édifices qu'étaient les palais de justice, dans lesquels devraient exercer les futurs magistrats. Par de subtiles transpositions, par le traitement des espaces ou des éléments qui les composent, Gillet parvint à insérer son bâtiment dans une désormais longue tradition architecturale judiciaire et dans un contexte patrimonial local fort. L'École nationale de la magistrature, établissement unique en son genre, ne prit corps que lentement, que ce soit dans son fonctionnement ou dans ses bâtiments, inaugurée seulement en 1972, douze ans après sa création. Bien qu'ayant fait l'objet d'agrandissements et de modifications par d'autres architectes, certains éminents comme Richard Rogers, l'édifice de Gillet peut encore se lire aujourd'hui à l'aune de son programme novateur et de son architecture remarquable.

Appointed in 1959 adviser to the Ministry of Justice, of which he was in reality the main architect for fifteen years, Guillaume Gillet (1912-1987) was called on to tackle two of the most important programmes mandated by the "garde des Sceaux" (French equivalent of "Lord Chancellor" or "Attorney General") Michel Debré: the construction of new prisons and the creation of a grande école for magistrates. Gillet is the author of at least ten centres of detention which renewed the design of prison, in its most functional aspect as well as in its aesthetics, thus following the principle of the "architecture parlante" (literally means "speaking architecture"), and rational architecture.

In 1960, in Bordeaux (Gironde department), Gillet, entrusted by the French National School for the Judiciary, was responsible for designing what from now on had to be the only place of 
education for all the magistrates of France, new institution planned in the judicial reform included in the French Constitution of 1958. This new function, the architect had to embody it within the buildings which contributed to giving a modern image of judiciary. However, the architect could not ignore the most significant features of the law courts buildings, in which the future magistrates would have to practice. By subtle transpositions, by the treatment of spaces or their composing elements, Gillet succeeded in inserting his building within a now long tradition of judicial architecture and within a strong local heritage context.

The French National School for the Judiciary, one-of-a-kind institution, took shape slowly, both in its functioning and in its buildings, and was only inaugurated in 1972, twelve years after its creation. Even though it has undergone enlargements and modifications made by other architects, some prominent ones like Richard Rogers, Gillet's building can still be interpreted today in the light of his innovative programme and his outstanding architecture.

\section{INDEX}

Mots-clés : architecture judiciaire, Gillet (Guillaume), École nationale de la magistrature, Fort du Hâ, salle des pas perdus, architecture parlante, architecture des Trente Glorieuses, architecture de la Ve République

Keywords : judicial architecture, Gillet (Guillaume), French National School for the Judiciary, Fort du Hâ, waiting room, architecture "parlante", architecture of the post-war reconstruction, architecture of the French Fifth Republic

\section{AUTEUR \\ FRANCK DELORME \\ Attaché de conservation, adjoint au directeur Cité de l'architecture et du patrimoine \\ franck.delorme@citedelarchitecture.fr}

